\title{
Efeito da restrição pré e pós-natal sobre o crescimento dos depósitos de gordura de cordeiros Santa Inês
}

\author{
[Effects of pre and postnatal nutritional restriction on internal fats growth of Santa Ines lambs] \\ L.C. Geraseev ${ }^{1}$, J.R.O. Perez ${ }^{2}$, F.A. Quintão ${ }^{3}$, B.C. Pedreira ${ }^{4}$, P.A. Carvalho ${ }^{3}$ \\ ${ }^{1}$ Instituto de Ciências Agrárias - UFMG \\ Av. Osmane Barbosa, $\mathrm{s} / \mathrm{n}$ \\ 39404-006 - Montes Claros, MG \\ ${ }^{2}$ Universidade Federal de Lavras - Lavras, MG \\ ${ }^{3}$ Aluno de pós-graduação - UFLA - Lavras, MG \\ ${ }^{4}$ Aluno de pós-graduação - ESALQ - Piracicaba, SP
}

\begin{abstract}
RESUMO
Avaliaram-se os efeitos da restrição pré e pós-natal sobre o tamanho e o crescimento dos depósitos de gordura de cordeiros Santa Inês. Foram utilizados 68 cordeiros, distribuídos em três tratamentos: com restrição pré-natal (RPRE), com restrição pós-natal (RPOS) e sem restrição (controle). Dentro de cada tratamento, os animais foram aleatoriamente sorteados para serem abatidos após o nascimento e com 10, $15,25,35$ e $45 \mathrm{~kg}$ de peso vivo. Os depósitos de gordura (omental, mesentérica, perirenal, pélvica e inguinal) foram registrados. Ao nascimento, os depósitos de gordura foram menores nos animais do tratamento RPRE. Entre 15 a $35 \mathrm{~kg}$ de peso, a quantidade de gordura foi igual nos animais dos três tratamentos e, a partir desse peso $(35 \mathrm{~kg})$, com exceção da gordura pélvica, os depósitos no animais do tratamento RPRE foram significativamente maiores. A restrição pré-natal elevou consideravelmente as taxas de ganho da gordura omental, mesentérica, perirenal e pélvica, enquanto a restrição pós-natal influenciou somente a taxa de ganho da gordura inguinal. Cordeiros de todos os grupos apresentaram taxas de desenvolvimento tardias $(\mathrm{b}>1)$.
\end{abstract}

Palavras-chave: ovino, crescimento alométrico, deposição de gordura, restrição alimentar

\begin{abstract}
Effects of pre and postnatal nutritional restriction on allometric growth of internal fat deposits in Santa Ines lambs were evaluated Sixty eight lambs were randomly alloted to three different nutritional restriction:prenatal nutritional restriction (PRER), postnatal nutritional restriction (POSR), and a control group without nutritional restriction. Lambs of each group were randomly sampled and slaughtered at birth and weighing 10,15,25, 35 and 45kg. Fat depositts (omental, mesenteric, perirenal, pelvic and inguinal) of slaughtered lambs were recorded. At birth fat deposits were small for animals from treatment RPRE. From 15 to $35 \mathrm{~kg}$ of live weight the amount of fat was similar for animals from all groups but lambs from PRER group, with live body weight heavier than $35 \mathrm{~kg}$, showed higher fat deposits except for pelvic fat. Prenatal nutritional restriction increased increased the omental, mesenteric, perirenal, and pelvic fat deposits and postnatal restriction decreased the inguinal fat deposits. Lambs from all groups showed a positive heterogenic development $(b>1)$.
\end{abstract}

Keywords: lamb, allometric growth, fat growth, alimentary restriction

Recebido em 11 de agosto de 2006

Aceito em 17 de maio de 2007

E-mail:lgeraseev@nca.ufmg.br 


\section{INTRODUÇÃO}

$\mathrm{Na}$ ovinocultura moderna, busca-se aumentar a deposição de proteína no tecido muscular, mantendo o conteúdo de gordura dentro do mínimo necessário, visando obter um produto de qualidade para o consumidor e, ao mesmo tempo, melhorar a eficiência econômica para o produtor. Para tanto, é necessário que se conheçam os processos de crescimento e desenvolvimento dos animais, inclusive das partes não componentes da carcaça.

No Brasil, nos últimos anos, houve um aumento significativo das pesquisas relacionadas com ovinos para produção de carne, entretanto pouca atenção foi dada ao desenvolvimento dos órgãos internos e dos depósitos de gordura.

Muitos fatores podem influenciar o crescimento desses depósitos; entre eles, destaca-se o nível nutricional. Várias pesquisas já mostraram a correlação existente entre o tamanho desses depósitos e o nível nutricional ao qual o animal é submetido (Rosa et al., 2002). Greenwood et al. (1998) afirmaram que animais submetidos à restrição alimentar pré-natal têm uma capacidade limitada de utilização da energia para deposição de tecidos magros (ossos e músculos). Quando suplementados adequadamente no período pósnatal, apresentam maior taxa de deposição de gordura na carcaça em comparação com os animais sem qualquer tipo de restrição.

Huidobro e Cañeque (1993) afirmaram que o estudo do desenvolvimento da gordura é muito importante, pois as proporções de cada depósito de gordura influenciam o valor comercial da carcaça. Prova disso são os principais sistemas de classificação de carcaça que utilizam medições sobre o tecido adiposo e os programas de seleção genética utilizados para diminuir o excesso de gordura nas carcaças, que estão baseados em medições da gordura subcutânea.

O objetivo deste trabalho foi estudar o efeito da restrição alimentar pré e pós-natal sobre o tamanho e o tipo de crescimento dos depósitos de gordura de cordeiros Santa Inês.

\section{MATERIAL E MÉTODOS}

O experimento foi realizado entre março de 2000 a novembro de 2001. Foram utilizados 68 cordeiros da raça Santa Inês, sendo 24 animais crias de ovelhas submetidas à restrição energética durante 0 terço final da gestação e 44 provenientes de ovelhas não submetidas à restrição alimentar durante a gestação. Neste segundo grupo, os animais foram distribuídos em dois subgrupos: um que recebeu alimentação pós-natal à vontade e outro que recebeu alimentação pós-natal restrita. Desse modo, foram identificados os efeitos da restrição pré e pós-natal.

Os tratamentos formaram três grupos de cordeiros: um grupo esteve sob restrição pré-natal, um esteve sob restrição pós-natal e um não foi submetido à restrição alimentar (controle). Dentro de cada grupo, os animais foram abatidos ao nascimento e quando atingiram $10,15,25,35$ e $45 \mathrm{~kg}$ de peso.

Ao nascer, os cordeiros permaneceram com suas mães durante três dias para mamar o colostro. Depois, foram separados e alojados em baias individuais, onde foram amamentados artificialmente até os 60 dias de idade.

Além do sucedâneo do leite, os animais tiveram acesso à dieta experimental, balanceada segundo as recomendações do ARC (The Nutrient..., 1980), fornecida duas vezes ao dia. Os animais dos grupos controle e restrição pré-natal receberam sucedâneo do leite à vontade e ração em quantidade para permitir uma sobra de $30 \%$ do total oferecido, enquanto os do grupo alimentação restrita receberam quantidades de sucedâneo e ração para proporcionar um ganho de $150 \mathrm{~g} /$ dia. A composição do sucedâneo e a da dieta experimental encontramse nas Tab. 1 e 2.

Tabela 1. Teores de proteína bruta (PB), extrato etéreo (EE), cálcio (Ca) e fósforo $(\mathrm{P})$ do sucedâneo usados para cordeiros

\begin{tabular}{lccccc}
\hline Ingrediente & $(\%)$ & PB & EE & Ca & P \\
\hline Leite de vaca & 70,0 & 2,33 & 2,72 & 0,09 & 0,07 \\
Leite em pó (varredura) & 5,0 & 1,44 & 2,46 & 0,02 & 0,03 \\
Ovo em pó & 5,0 & 2,08 & 2,57 & 0,06 & 0,04 \\
Água & 20,0 & - & - & - & - \\
Total (Sucedâneo) & 100,00 & 5,85 & 7,74 & 0,17 & 0,14 \\
\hline
\end{tabular}


Tabela 2. Teores de matéria seca (MS), proteína bruta (PB), fibra em detergente neutro (FDN), fibra em detergente ácido (FDA), cálcio (Ca) e fósforo (P) dos ingredientes da dieta, expressos em porcentagem da matéria seca, usados para cordeiros

\begin{tabular}{|c|c|c|c|c|c|c|}
\hline Ingredientes & $\begin{array}{l}\text { MS } \\
(\%)\end{array}$ & $\begin{array}{l}\mathrm{PB} \\
(\%)\end{array}$ & $\begin{array}{c}\text { FDN } \\
(\%)\end{array}$ & $\begin{array}{c}\text { FDA } \\
(\%)\end{array}$ & $\begin{array}{l}\mathrm{Ca} \\
(\%)\end{array}$ & $\begin{array}{c}\mathrm{P} \\
(\%)\end{array}$ \\
\hline $\begin{array}{lll}\text { Feno } & \text { de } & \text { Coast } \\
\text { cross } & & \\
\end{array}$ & 91,30 & 8,53 & 78,63 & 33,93 & 0,733 & 0,434 \\
\hline Milho grão & 86,77 & 10,56 & 21,58 & 4,03 & 0,063 & 0,311 \\
\hline Farelo de soja & 88,40 & 45,62 & 20,70 & 10,17 & 0,452 & 0,781 \\
\hline Calcário calcítico & 99,99 & - & - & - & 35,84 & - \\
\hline Sal comum & 99,82 & - & - & - & - & - \\
\hline Supl. min./vit. ${ }^{1}$ & 94,36 & - & - & - & 23,00 & 9,00 \\
\hline
\end{tabular}

${ }^{1}$ Nutrientes $/ \mathrm{kg}$ de suplemento: Cálcio $=230 \mathrm{~g}$; Fósforo $=90 \mathrm{~g}$; Enxofre $=15 \mathrm{~g}$; Magnésio $=20 \mathrm{~g}$; Sódio $=48 \mathrm{~g}$; Cobalto $=$ $100 \mathrm{mg} ;$ Cobre $=700 \mathrm{mg} ;$ Ferro $=2.000 \mathrm{mg} ;$ Iodo $=80 \mathrm{mg} ;$ Manganês $=1250 \mathrm{mg} ;$ Selênio $=200 \mathrm{mg} ;$ Zinco $=2.700 \mathrm{mg}$; Flúor=900mg; Vitamina A=200.000UI, Vitamina D3=60.000UI; Vitamina E=60UI.

Os animais foram submetidos a 16 horas de jejum, com acesso à água antes de serem abatidos. Ao término desse período, foi realizada pesagem para determinação do peso ao abate.

O abate foi feito por sangramento mediante corte da carótida e jugular. Após o abate, foram retirados e pesados os depósitos de gordura omental e mesentérica. Concluída a evisceração e a retirada da cabeça, pés, cauda e testículos, obteve-se a carcaça inteira que foi levada à câmara fria com temperatura de $2^{\circ} \mathrm{C}$ por 24 horas. Após esse período, foram retirados e pesados os depósitos de gordura perirrenal, pélvica e inguinal.

O período experimental não teve duração préfixada, pois correspondeu ao período necessário para que os animais atingissem o peso prédeterminado para o abate.

Para a análise do tamanho do depósito de gordura, foi utilizado o delineamento experimental inteiramente ao acaso, em esquema fatorial 3x6 (três tratamentos: controle, restrição pré e restrição pós-natal, e seis pesos de abate: ao nascimento, e aos $10,15,25,35$ e $45 \mathrm{~kg}$ ) com quatro repetições, em que cada animal representou uma unidade experimental. Essas variáveis foram analisadas utilizando o modelo estatístico abaixo e as médias foram comparadas por meio do teste Scott-Knott.

$Y_{i j k}=\mu+\alpha_{i}+\beta_{j}+(\alpha \beta)_{i j}+\xi_{(i j) k}$, em que:

$\mathrm{Y}_{\mathrm{ijk}}$ são os valores observados para as variáveis supracitadas no nível i de tratamentos de restrição e no nível $\mathrm{j}$ de peso de abate, na repetição $\mathrm{k}(\mathrm{k}=1,2,3,4)$;

$\mu$ é a média geral; $\alpha_{\mathrm{i}}$ é o efeito do nível $\mathrm{i}$ de tratamento ( $\left.\mathrm{i}=1,2,3\right)$;

$\beta_{\mathrm{j}}$ é o efeito do nível $\mathrm{j}$ do peso ao abate $(j=1,2,3,4,5,6)$;

$(\alpha \beta)_{i j}$ é o efeito da interação dos fatores tratamento e peso de abate;

$\xi_{(\mathrm{ij}) \mathrm{k}}$ é o erro experimental associado à observação $\mathrm{Y}_{\mathrm{ijk}}$, que por hipótese tem distribuição normal com média zero e variância $\sigma^{2}$.

O desenvolvimento dos depósitos de gordura omental, mesentérica, perirenal, pélvica e inguinal foi avaliado segundo estudo alométrico, correlacionando o crescimento de cada depósito com o crescimento do corpo vazio (PCV), utilizando-se a equação potência $\mathrm{Y}=\mathrm{aX} \mathrm{X}^{\mathrm{b}}$ (Huxley, 1932), linearizada mediante transformação logarítmica $(\log \mathrm{Y}=\operatorname{loga}+\mathrm{b} \log \mathrm{X})$. Para $\mathrm{a}$ verificação da hipótese $b=1$, foi utilizado o teste t. O modelo estatístico utilizado neste caso foi: $Y_{i j}=\mu+b_{1} x_{1 i}+e_{i j}$, em que:

$Y_{i j}$ são os valores observados para o logaritmo do peso dos depósitos de gordura do animal no nível i de tratamentos de restrição, na repetição j;

$\mu$ é a média geral;

$\mathrm{b}_{1}$ é o coeficiente de regressão;

$\mathrm{x}_{1 \mathrm{i}}$ é logaritmo do peso corporal vazio do animal; $\mathrm{e}_{\mathrm{ij}}$ é o erro experimental associado à observação $\mathrm{Y}_{\mathrm{ij}}$, que por hipótese tem distribuição normal com média zero e variância $\sigma^{2}$.

As taxas de ganho dos depósitos (g) por quilograma de ganho de peso corporal vazio foram obtidas a partir da derivação das equações de crescimento alométrico, obtendo-se equações do tipo $\mathrm{Y}^{\prime}=\mathrm{b} \cdot 10^{\mathrm{a}} . \mathrm{X}^{(\mathrm{b}-1)}$. 


\section{RESULTADOS E DISCUSSÃO}

Na Tab. 3, são apresentados os tamanhos (g) dos diferentes depósitos de gordura dos cordeiros. Ao nascimento, a restrição alimentar pré-natal diminuiu o tamanho de todos os depósitos de gordura, atribuído ao suprimento limitado de nutrientes durante o período pré-natal, o que impediu que os animais armazenassem energia na forma de tecido adiposo. Contudo, já aos $15 \mathrm{~kg}$, o tamanho dos depósitos de gordura foi semelhante ao dos grupos controle e restrição pós-natal. Aos $45 \mathrm{~kg}$, com exceção da gordura pélvica, o tamanho dos depósitos nos animais da RPRE foi significativamente maior que o dos animais dos outros grupos, demonstrando que houve maior deposição de gordura interna após o nascimento.

Tabela 3. Tamanhos médios (g) dos depósitos de gordura de cordeiros Santa Inês ao nascimento e aos 15 e $45 \mathrm{~kg}$ de peso, submetidos a diferentes manejos alimentares

\begin{tabular}{|c|c|c|c|}
\hline Peso & Controle & Restrição pré-natal & Restrição pós-natal \\
\hline \multicolumn{4}{|c|}{ Omental } \\
\hline Nasc & $9 a$ & $2 b$ & $9 \mathrm{a}$ \\
\hline 15 & $60 \mathrm{a}$ & $93 a$ & $86 a$ \\
\hline 45 & $821 \mathrm{~b}$ & $1.380 \mathrm{a}$ & $758 b$ \\
\hline Média & $306 \mathrm{~b}$ & $402 a$ & $297 \mathrm{~b}$ \\
\hline \multicolumn{4}{|c|}{ Mesentérica } \\
\hline Nasc & $16 \mathrm{a}$ & $5 \mathrm{~b}$ & $16 \mathrm{a}$ \\
\hline 15 & $151 \mathrm{a}$ & $149 \mathrm{a}$ & $171 \mathrm{a}$ \\
\hline 45 & $563 \mathrm{~b}$ & $892 a$ & $632 \mathrm{~b}$ \\
\hline Média & $260 \mathrm{~b}$ & $310 \mathrm{a}$ & $274 b$ \\
\hline \multicolumn{4}{|c|}{ Perirrenal } \\
\hline Nasc & $26 a$ & $1 b$ & $26 a$ \\
\hline 15 & $42 \mathrm{a}$ & $84 a$ & $58 \mathrm{a}$ \\
\hline 45 & $410 \mathrm{~b}$ & $661 \mathrm{a}$ & $357 \mathrm{~b}$ \\
\hline Média & $174 b$ & $230 \mathrm{a}$ & $163 b$ \\
\hline \multicolumn{4}{|c|}{ Pélvica } \\
\hline Nasc & $7 \mathrm{a}$ & $1 b$ & $7 \mathrm{a}$ \\
\hline 15 & $22 \mathrm{a}$ & $32 \mathrm{a}$ & $25 \mathrm{a}$ \\
\hline 45 & $140 \mathrm{a}$ & $155 \mathrm{a}$ & $125 \mathrm{a}$ \\
\hline Média & $53 b$ & $64 \mathrm{a}$ & $49 b$ \\
\hline \multicolumn{4}{|c|}{ Inguinal } \\
\hline Nasc & $3 a$ & $1 b$ & $3 a$ \\
\hline 15 & $21 \mathrm{a}$ & $25 \mathrm{a}$ & $19 a$ \\
\hline 45 & $178 \mathrm{a}$ & $184 a$ & $136 \mathrm{~b}$ \\
\hline Média & $69 \mathrm{a}$ & $75 \mathrm{a}$ & $54 \mathrm{~b}$ \\
\hline
\end{tabular}

Médias seguidas de letras distintas na linha diferem entre si pelo teste Scott-Knott $(\mathrm{P}<0,05)$.

Segundo Greenwood et al. (1998), a maior deposição de gordura apresentada por animais sob restrição pré-natal pode ser explicada pela alteração que ocorre no seu metabolismo energético. Esses animais têm menor exigência energética de mantença e, quando suplementados adequadamente no período pós-natal, apresentam maior taxa de deposição de gordura na carcaça em comparação com animais sem qualquer tipo de restrição. Ainda segundo os autores, essa maior taxa de deposição do tecido adiposo pode ser atribuída à capacidade limitada dos tecidos magros (ossos e músculos) de responderem à suplementação alimentar.
Quanto aos efeitos da restrição pós-natal, os dados revelam que somente a gordura inguinal foi afetada. Embora vários trabalhos (Hood e Thornton, 1980; Perón et al., 1993; Fluharty e McClure, 1997) tenham demonstrado que a atividade lipogênica é menor em animais submetidos a esse tipo de restrição, sabe-se que essa resposta depende do nível da restrição imposta. Notter et al. (1983), ao avaliarem os efeitos de quatro níveis de ingestão sobre o crescimento alométrico de cordeiros Dorset e Finnish Landrace, concluíram que as restrições de $85 \%$ e $70 \%$ em relação à não restrição não alteraram a porcentagem de gordura corporal, e 
que os animais alimentados só para mantença apresentaram redução significativa na gordura corporal. Fluharty e McClure (1997) verificaram que a restrição de $85 \%$ resultou em menos gordura visceral que a alimentação à vontade.

Segundo Hood e Thornton (1980), fatores como nível nutricional, raça, sexo e idade influenciaram o nível de atividade lipogênica em ovinos. Neste experimento, os animais submetidos à restrição pós-natal foram abatidos, em média, com 174 dias, enquanto os animais- controle o foram com 124 dias. Essa diferença na idade de abate pode ter contribuído para a semelhança nos tamanhos dos depósitos de gordura omental, mesentérica, perirenal e pélvica.

A partir do peso corporal vazio e do peso dos depósitos de gordura, foram determinadas equações de regressão que permitiram estimar o crescimento de cada depósito em relação ao peso corporal vazio. Essas equações alométricas são apresentadas na Tab. 4.

Tabela 4. Equações de alometria para os depósitos de gordura em relação ao peso corporal vazio (PCV) de cordeiros Santa Inês submetidos a diferentes manejos alimentares

\begin{tabular}{|c|c|c|c|}
\hline Tratamento & Equação & Teste $\mathrm{t}$ & $\mathrm{R}^{2}$ \\
\hline \multicolumn{4}{|c|}{ Omental } \\
\hline Controle & $\begin{array}{l}\log \mathrm{OME}=-6,4358+2,0344 \log \\
\text { PCV } \\
\log \mathrm{OME}=-7,5060+2,2950 \log \\
\text { PCV }\end{array}$ & $b>1$ & 0,9557 \\
\hline RPRE & $\log \mathrm{OME}=-6,4275+2,0286 \log$ & $b>1$ & 0,9640 \\
\hline RPOS & PCV & $\mathrm{b}>1$ & 0,9519 \\
\hline \multicolumn{4}{|c|}{ Mesentérica } \\
\hline Controle & $\begin{array}{l}\log \text { MES }=-4,4058+1,5734 \log \\
\text { PCV } \\
\log M E S=-5,4308+1,8224 \log \end{array}$ & $b>1$ & 0,9604 \\
\hline RPRE & $\begin{array}{l}\text { PCV } \\
\log \text { MES = -4,2997 + 1,5535 log }\end{array}$ & $b>1$ & 0,9193 \\
\hline RPOS & PCV & $\mathrm{b}>1$ & 0,9587 \\
\hline \multicolumn{4}{|c|}{ Perirenal } \\
\hline Controle & $\begin{array}{l}\log \text { PER }=-3,3170+1,2718 \log \\
\text { PCV } \\
\text { Log PER }=-7,9124+2,3385 \log \end{array}$ & $b>1$ & 0,8115 \\
\hline RPRE & $\begin{array}{l}\text { PCV } \\
\log \text { PER }=-3,4976+1,3033 \log \end{array}$ & $b>1$ & 0,8987 \\
\hline RPOS & PCV & $b>1$ & 0,7623 \\
\hline \multicolumn{4}{|c|}{ Pélvica } \\
\hline Controle & $\begin{array}{l}\log \text { PEL }=-3,6505+1,2379 \log \\
\text { PCV } \\
\text { Log PEL }=-6,2211+1,8372 \log \end{array}$ & $b>1$ & 0,8754 \\
\hline RPRE & $\begin{array}{l}\text { PCV } \\
\text { Log PEL }=-4,0246+1,3089 \log \end{array}$ & $b>1$ & 0,9018 \\
\hline RPOS & PCV & $b>1$ & 0,8565 \\
\hline \multicolumn{4}{|c|}{ Inguinal } \\
\hline Controle & $\begin{array}{l}\log I N G=-5,9047+1,7762 \log \\
P C V \\
\log I N G=-6,6053+1,9368 \log \end{array}$ & $b>1$ & 0,9272 \\
\hline RPRE & $\begin{array}{l}\text { PCV } \\
\log I N G=-5,5540+1,6718 \log \end{array}$ & $b>1$ & 0,9055 \\
\hline RPOS & $\mathrm{PCV}$ & $\mathrm{b}>1$ & 0,9575 \\
\hline
\end{tabular}

$\mathrm{RPRE}=$ com restriçãopré-natal; RPOS=com restrição pós-natal. 
Os coeficientes de determinação encontrados para as equações de regressão mostram que houve um ajuste adequado dos dados, com pouca dispersão. Os coeficientes de regressão "b" foram significativos $(\mathrm{P}<0,01)$ para todos os manejos alimentares. Em todos os depósitos de gordura verificou-se crescimento heteregônico positivo.

Rosa et al. (2002), ao avaliarem o crescimento dos depósitos de gordura interna e perirenal de cordeiros Texel, concluíram que o desenvolvimento da gordura interna foi tardio $(b=1,35)$ e o da gordura perirenal, isogônico $(b=0,869)$.

Santos (2002), ao trabalhar com cordeiros Santa Inês, verificou que o desenvolvimento dos depósitos de gordura omental, mesentérica, perirenal e cavitária foi tardio, com intensidades diferentes de crescimento, sendo, em ordem crescente, cavitária $(b=1,26)$, perirenal $(b=1,80)$, mesentérica $(b=1,53)$ e omental $(b=2,32)$. Esse mesmo comportamento foi observado para os depósitos de gordura omental, mesentérica, perirenal e pélvica do presente experimento.

A restrição pré-natal não alterou o tipo de desenvolvimento dos depósitos de gordura. Não se observou efeito da restrição pós-natal devido ao baixo nível da restrição imposta.

Rosa et al. (2002), ao avaliarem o crescimento do tecido adiposo em cordeiros submetidos a diferentes métodos de alimentação, verificaram que o desenvolvimento do tecido adiposo foi tardio e não houve efeito do método de alimentação sobre o comportamento do crescimento desse tecido.

$\mathrm{Na}$ Tab. 5, são apresentadas as taxas de ganho ( $\mathrm{g} / \mathrm{kg}$ de peso corporal vazio) dos diferentes depósitos de gordura.

Tabela 5. Taxas de crescimento ( $\mathrm{g} / \mathrm{kg}$ de peso corporal vazio) dos depósitos de gordura de cordeiros Santa Inês ao nascimento e aos 15 e $45 \mathrm{~kg}$ de peso, submetidos a diferentes manejos alimentares

\begin{tabular}{|c|c|c|c|}
\hline Peso & Controle & Restrição pré-natal & Restrição pós-natal \\
\hline \multicolumn{4}{|c|}{ Omental } \\
\hline Nasc & 4,20 & 3,66 & 4,24 \\
\hline 15 & 13,09 & 15,17 & 13,12 \\
\hline 45 & 40,78 & 62,94 & 40,62 \\
\hline Média & 20,05 & 27,60 & 20,03 \\
\hline \multicolumn{4}{|c|}{ Mesentérica } \\
\hline Nasc & 7,42 & 6,61 & 8,09 \\
\hline 15 & 13,92 & 16,31 & 14,87 \\
\hline 45 & 26,14 & 40,25 & 27,31 \\
\hline Média & 16,64 & 22,06 & 17,61 \\
\hline \multicolumn{4}{|c|}{ Perirrenal } \\
\hline Nasc & 5,93 & 2,10 & 5,28 \\
\hline 15 & 7,99 & 9,16 & 7,37 \\
\hline 45 & 10,77 & 39,84 & 10,28 \\
\hline Média & 8,51 & 17,17 & 7,93 \\
\hline \multicolumn{4}{|c|}{ Pélvica } \\
\hline Nasc & 2,02 & 1,22 & 1,65 \\
\hline 15 & 2,62 & 3,06 & 2,32 \\
\hline 45 & 3,40 & 7,69 & 3,25 \\
\hline Média & 2,76 & 4,18 & 2,50 \\
\hline \multicolumn{4}{|c|}{ Inguinal } \\
\hline Nasc & 1,44 & 1,22 & 1,31 \\
\hline 15 & 3,39 & 3,43 & 2,74 \\
\hline 45 & 7,94 & 9,59 & 5,72 \\
\hline Média & 4,47 & 4,95 & 3,42 \\
\hline
\end{tabular}

Ao nascimento, as taxas de ganho dos depósitos de gordura foram menores para os animais da restrição pré-natal, entretanto, com $15 \mathrm{~kg}$, esses animais já apresentavam taxas de crescimento semelhantes ou mais altas que os animais controle e da restrição pós-natal. Aos $45 \mathrm{~kg}$, essas taxas eram maiores para os animais RPRE, demonstrando que esses animais apresentavam 
maior deposição de gordura interna após o nascimento. Essa maior deposição de gordura apresentada pelos animais da restrição pré-natal pode ser explicada pela alteração ocorrida no seu metabolismo energético, levando-os a depositarem mais gordura devido à capacidade limitada de seus tecidos magros (ossos e músculos) em responderem à suplementação alimentar.

\section{CONCLUSÕES}

A restrição pré-natal alterou o tamanho dos depósitos de gordura de cordeiros Santa Inês, aumentando as taxas de crescimento desses depósitos. Essa maior deposição de gordura eleva os custos com o arraçoamento destes animais, o que ressalta a importância da suplementação das ovelhas gestantes no terço final de gestação. Já a restrição pós-natal alterou somente a taxa de crescimento da gordura inguinal, com redução no tamanho deste depósito.

\section{REFERÊNCIAS BIBLIOGRÁFICAS}

FLUHARTY, F.L.; McCLURE, K.E. Effects of dietary energy intake and protein concentration on performance and visceral organ mass in lambs. J. Anim. Sci., v.75, p.604-610, 1997.

GREENWOOD, P.L.; HUNT, A.S.; HERMANSON, J.W. et al. Effects of birth weight and postnatal nutrition on neonatal sheep: I. Body growth and composition, and some aspects of energetic efficiency. J. Anim. Sci., v.76, p.2354-2367, 1998.
HOOD, R.L.; THORNTON, R.F. The effect of compensatory growth on lipogenesis in ovine carcass adipose tissue. Aust. J. Agric. Res., v.31, p.155-161, 1980.

HUIDOBRO, F.R.; CAÑEQUE, V. Producción de carne en corderos de raza Manchega. II. Conformación y estado de engrasamiento de la canal y proporción de piezas en distintos tipos comerciales. Invest. Agr.: Prod. Sanid. Anim., v.8, p.233-243, 1993.

HUXLEY, J.S. Problems of relative growth. London: Methuen. 1932.

NOTTER, D.R.; FERREL, C.L.; FIELD, R.A. Effects of breed and intake level on allometric growth patterns in ram lambs. J. Anim. Sci., v.56, p.380-395, 1983

PERON, A.J.; FONTES, C.A.A.; LANA, R.P. et al. Tamanho dos órgãos internos e distribuição da gordura corporal em novilhos de cinco grupos genéticos, submetidos à alimentação restrita e ad libitum. Rev. Soc. Bras. Zootec., v.22, p.813-819, 1993.

ROSA, G.T.; PIRES, C.C.; SILVA, J.H.S. et al. Proporções e coeficientes de crescimento dos não-componentes da carcaça de cordeiros e cordeiras em diferentes métodos de alimentação. Rev. Soc. Bras. Zootec., v.31, p.2290-2298, 2002.

SANTOS, C. L. Estudo do crescimento e da composição química dos cortes da carcaça de cordeiros Santa Inês e Bergamácia. 2002. 257f. Tese (Doutorado em Zootecnia) - Universidade Federal de Lavras, Lavras, MG.

THE NUTRIENT requirements of farm animals, London: ARC, 1980. 351p. 